\title{
Variabilité des utilisations de Diospyros mespiliformis Hochst. suivant les facteurs sociodémographiques au Nord-Bénin
}

Alain DAANON ${ }^{1}$

Elie Antoine PADONOU ${ }^{2}$

Bokon Alexis AKAKPO ${ }^{1,3}$

Marcel HOUINATO ${ }^{1}$

${ }^{1}$ Université d'Abomey-Calavi Faculté des sciences agronomiques Laboratoire d'écologie appliquée 01 BP 526, Cotonou

Bénin

${ }^{2}$ Université nationale d'agriculture École de foresterie tropicale BP 43, Kétou

Bénin

${ }^{3}$ WASCAL (West African Science Service Centre on Climate Change and Adapted Land Use) Federal University of Technology PMB 65 Minna, Niger State Nigeria

Auteur correspondant / Corresponding author: Bokon Alexis AKAKPO abakakpo@yahoo.fr

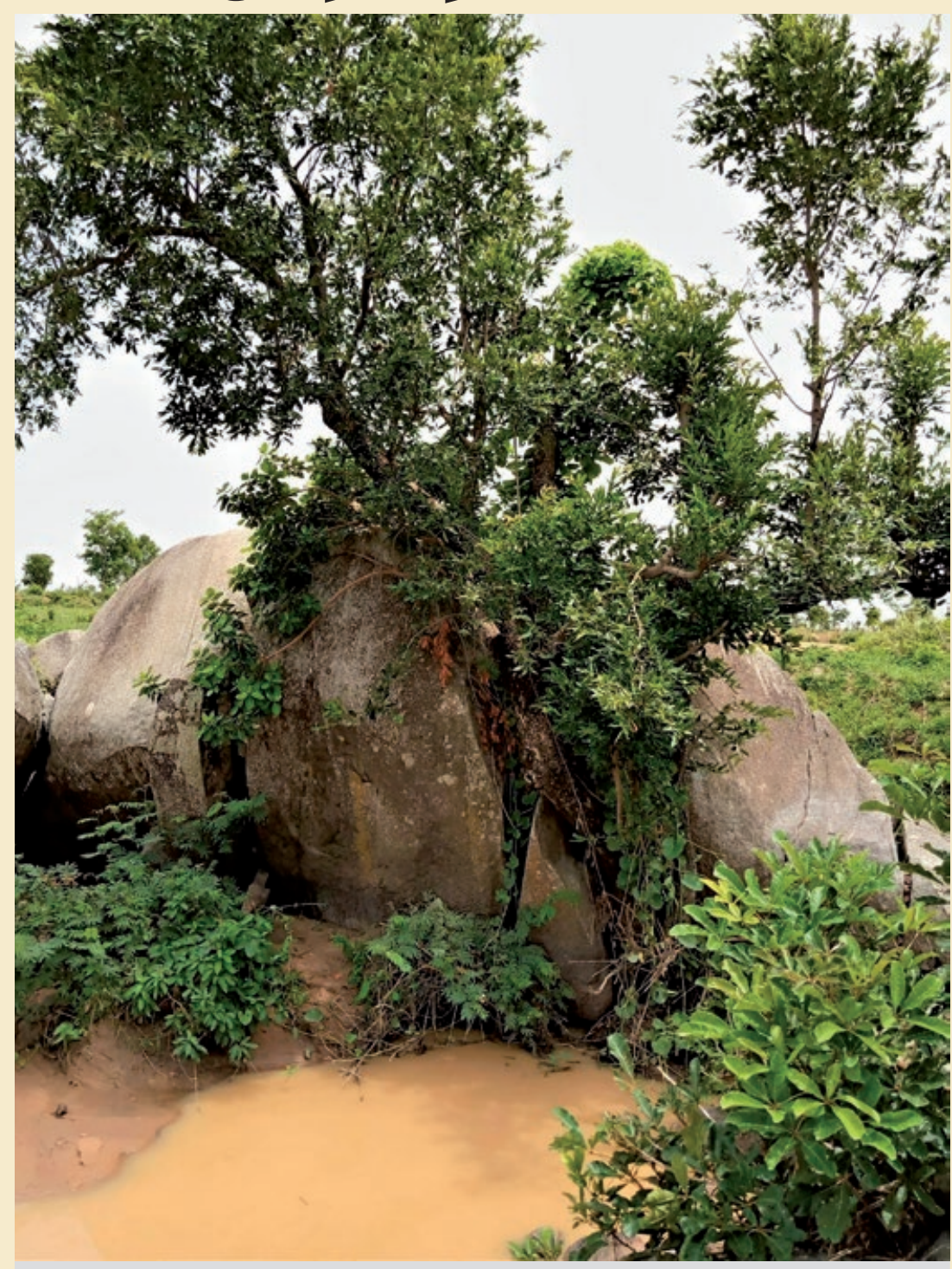

Photo 1.

Arbre de Diospyros mespiliformis en peuplement naturel.

Tree of Diospyros mespiliformis in a natural stand.

Photo A. Daanon.

\section{Ccirad ata}

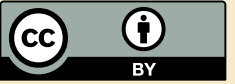

Citer l'article / To cite the article

Daanon A., Padonou E. A., Akakpo B. A., Houinato M., 2021. Variabilité des utilisations de Diospyros mespiliformis Hochst. suivant les facteurs sociodémographiques au Nord-Bénin. Bois et Forêts des Tropiques, 347: 29-40. Doi : https://doi.org/10.19182/bft2021.347.a36344 


\section{RÉSUMÉ}

Variabilité des utilisations de Diospyros mespiliformis Hochst. suivant les facteurs sociodémographiques au Nord-Bénin

Diospyros mespiliformis est une espèce d'arbre très peu étudiée malgré son importance pour les communautés locales au Bénin. Cette étude a évalué la variabilité des usages de l'espèce et de ses organes suivant les facteurs sociodémographiques (la provenance, les catégories socioculturelles, le sexe, l'âge et la profession des utilisateurs). Une enquête auprès de 233 participants a été réalisée dans les communes de Tchaourou, Sinendé et Bembèrèkè. La fréquence de citation, les usages rapportés et l'indice d'importance des organes de l'espèce ont été calculés. Des analyses de la covariance sur les usages totaux rapportés ont été réalisées afin d'évaluer les déterminants des usages de l'espèce. Des analyses en composantes principales ont été effectuées afin de caractériser les usages, suivant les déterminants des usages de l'espèce. Les résultats ont révélé que le sexe, l'âge et la provenance socioculturelle $(P<0,05)$ ont déterminé les usages dans la zone d'étude. Les hommes adultes ont eu plus d'intérêt pour l'espèce. Les Peuhls de Tchaourou et les Bariba de Sinendé ont utilisé davantage l'espèce comme bois énergie et bois d'œuvre. Les Bariba de Tchaourou, de Sinendé et les Peuhls de Sinendé sont plus intéressés par l'espèce pour des usages alimentaires, le fourrage et la pharmacopée. L'utilisation des organes est déterminée par l'âge et les interactions provenance-profession-âge $(P<0,05)$. Les racines et les écorces sont les parties les plus appréciées par les adultes et les personnes âgées. Les éleveurs, en général, et les agriculteurs de Tchaourou sont particulièrement intéressés par les racines, les tiges et les noix de l'espèce. Les agriculteurs de Bembèrèkè et les commerçants de Sinendé et de Bembèrèkè sont, quant à eux, plus enclins à l'usage des écorces. Par ailleurs, les usages de l'espèce en tant que bois et fourrage ont affecté négativement $(P<0,000)$ son usage alimentaire. Ces résultats sont à prendre en compte pour une stratégie de valorisation et de conservation durable de l'espèce.

Mots-clés : Diospyros mespiliformis, importance ethnobotanique, variabilité des usages, Nord-Bénin.

\section{ABSTRACT}

\section{Variability in uses of Diospyros mespiliformis Hochst. according to socio- demographic factors in northern Benin}

Diospyros mespiliformis is a tree species that has been very little studied despite its importance for local communities in Benin. This study assessed the variability of uses of the species and its organs according to socio-economic factors (origin, socio-cultural background and categories, gender, age and professions). A survey among 233 participants was conducted in the municipalities of Tchaourou, Sinendé and Bembèrèkè. Citation frequencies, reported uses and the index of importance of the different organs were calculated. Covariance analyses of all reported uses were performed to assess the factors determining uses of the species. Principal Component Analyses were performed to characterise these uses according to the determinants of species use. The results show that in the study zone, uses were determined by gender, age and socio-cultural background $(P<0.05)$. Adult men had more interest than others in the species. The Fulani from Tchaourou and the Bariba from Sinendé used the species most for fuel and timber. The Bariba from Tchaourou and Sinendé and the Fulani from Sinendé had more interest in the species for food, fodder and medicinal uses. Uses of the different organs were determined by age and origin-profession-age interactions $(P<0.05)$. The roots and bark were most used by adults and elderly people. Herders in general and the farmers from Tchaourou were interested more specifically in the roots, stems and fruits of the species. The farmers from Bembèrèkè and traders from Sinendé and Bembèrèkè had a greater tendency to use the bark. Furthermore, uses of the species for its wood and for fodder had a negative effect $(P<0.000)$ on its uses for food. These results should be taken into account in designing a strategy for the sustainable use and conservation of the species.

\section{Keywords: Diospyros mespiliformis,} ethnobotanical importance, variability of uses, northern Benin.

\section{HOUINATO}

\section{RESUMEN}

\section{Variabilidad de los usos del Diospyros mespiliformis Hochst. según los factores sociodemográficos en el norte de Benín}

Diospyros mespiliformis es una especie de árbol muy poco estudiada a pesar de su importancia para las comunidades locales de Benín. Este estudio evaluó la variabilidad de los usos de la especie y de sus órganos según los factores sociodemográficos (origen, categorías socioculturales, sexo, edad y profesión de los usuarios). Se realizó una encuesta a 233 participantes en los municipios de Tchaourou, Sinendé y Bembèrèkè. Se calcularon la frecuencia de citaciones, los usos citados y el índice de importancia de los órganos de la especie. Se efectuaron análisis de la covarianza sobre los usos totales citados para evaluar los factores determinantes en los usos de la especie. Se realizaron análisis de componentes principales para caracterizar los usos, según los determinantes de los usos de la especie. Los resultados revelaron que el sexo, la edad y el origen sociocultural $(P<0,05)$ determinaron los usos en la zona de estudio. Los hombres adultos tenían más interés por la especie. Los Peuhl de Tchaourou y los Bariba de Sinendé utilizaban más la especie como leña y madera de construcción. Los Bariba de Tchaourou, de Sinendé y los Peuhl de Sinendé están más interesados por la especie para usos alimentarios, forraje y farmacopea. El uso de órganos viene determinado por la edad y las interacciones origen-profesión-edad $(P<0,05)$. Las raíces y las cortezas son las partes más utilizadas por los adultos y los ancianos. Los ganaderos, en general, y los agricultores de Tchaourou están especialmente interesados en las raíces, los tallos y las nueces de la especie. Los agricultores de Bembèrèkè y los comerciantes de Sinendé y de Bembèrèkè están más orientados al uso de las cortezas. Asimismo, los usos de la especie como madera y forraje afectaron negativamente $(P<0,000)$ su utilización alimentaria. Hay que tener en cuenta estos resultados para elaborar una estrategia de valorización y de conservación sostenible de la especie.

Palabras clave: Diospyros mespiliformis, importancia etnobotánica, variabilidad de usos, norte de Benín. 


\section{Introduction}

Les fruitiers sauvages jouent un rôle prépondérant dans le quotidien des communautés rurales. De nos jours, ils occupent une place importante dans les industries agroalimentaires et pharmaceutiques à travers le monde (Mapongmetsem et al., 2012). Ils sont utilisés à des fins de consommation directe, commerciales et médicinales (Idohou et al., 2013 ; Maxted et al., 2008). Ce sont des compléments alimentaires pendant les périodes de soudure (Diarra et al., 2016). Durant ces dernières années, au Bénin, de nombreuses études ont été effectuées concernant les diverses contributions que peuvent apporter les fruitiers sauvages. C'est le cas, par exemple, de leur participation dans l'alimentation des populations locales (Lougbegnon et al., 2015 ; Akouehou et al., 2014 ; Fandohan et al., 2010), la diversité de leurs produits (Assogbadjo et al., 2009 ; Codjia et al., 2003), la diversité des usages et l'importance socioculturelle et socio-économique de leurs différents constituants (Gouwakinnou et al., 2011 ; Koura et al., 2011 ; Ekué et al., 2010 ; Fandohan et al., 2010 ; Vodouhê et al., 2009). Toutefois, très peu d'études ont été consacrées à Diospyros mespiliformis Hochst.

Diospyros mespiliformis Hochst. est un fruitier sauvage de la famille des Ebenaceae encore appelé ébénier d'Afrique (photo 1). Il est relativement négligé scientifiquement au Bénin, mais très fortement convoité dans plusieurs localités voisines pour plusieurs usages (Dossou-Yovo et al., 2014 ; Djagoun et al., 2010). Les individus de l'espèce se retrouvent en abondance dans les régions tropicales d'Afrique ( $\mathrm{du}$

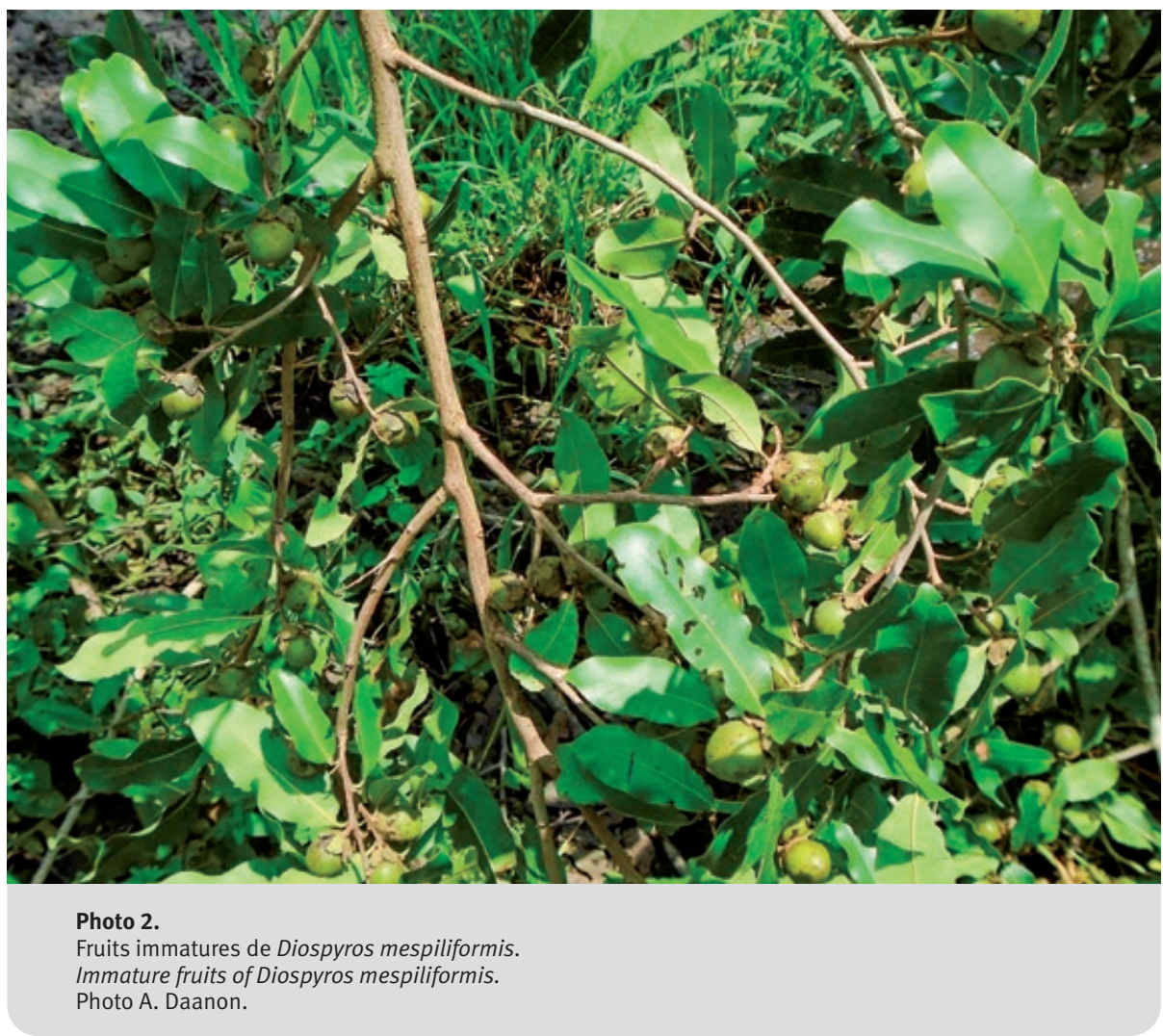

Sénégal au Cameroun). D’après Bonnet et al. (2008), les arbres adultes de $D$. mespiliformis peuvent atteindre $30 \mathrm{~m}$ de hauteur. Son habitat varie généralement des forêts guinéennes, des galeries forestières à des bords de rivière, sur des termitières et des collines rocheuses. L'espèce se rencontre aussi sur les sols lourds et bien drainés (Arbonnier, 2002). C'est une espèce d'arbre à fragments de fleurs blanches (photo 2) qui produit des fruits dont la pulpe est très sucrée et très appréciée non seulement par les singes et les oiseaux, qui assurent sa dispersion en milieu naturel, mais aussi par les populations rurales (Arbonnier, 2002).

L'ébénier d'Afrique présente un très grand intérêt pour les différentes catégories sociodémographiques dans le Nord du Bénin (Vodouhê et al., 2009). En effet, il est intensivement utilisé en alimentation, en médicine, en tant que bois de service et d'œuvre, en pratiques culturelles et autres (Vodouhê et al., 2009). Cependant, malgré l'importance révélée de l'espèce pour les communautés locales, les connaissances restent faibles sur ses différentes utilisations (variabilité des utilisations suivant les caractéristiques sociodémographiques, organes récoltés, et interactions entre les différentes utilisations) possibles (Avocèvou-Ayisso et al., 2012). Ainsi, il est très important de documenter les informations relatives à l'espèce sur ces différents aspects afin de réussir les stratégies de sa valorisation et gestion durable. Par ailleurs, en se basant sur des études antérieures, nous pouvons supposer que les utilisations et les parties collectées des espèces de fruitiers sauvages diffèrent non seulement d'une catégorie socioculturelle à l'autre mais aussi entre les utilisateurs d'une même catégorie socioculturelle ne se retrouvant pas sur le même territoire. De même, un type d'utilisation peut influencer la possibilité d'utiliser l'espèce à d'autres fins (Avocèvou-Ayisso et al., 2012). D’où les objectifs visés dans cette étude, consistant à caractériser les utilisations de $D$. mespiliformis suivant les différentes catégories sociodémographiques du milieu d'étude, à évaluer les usages ethnobotaniques des organes de l'espèce suivant ces différentes catégories sociodémographiques, et enfin à estimer l'influence des catégories d'utilisation sur le niveau d'usage alimentaire de l'espèce. 


\section{Méthodes}

\section{Milieu d'étude et choix des localités d'enquête}

Les enquêtes ont été effectuées dans les localités riveraines des forêts classées de Wari-Maro, Alibori Supérieur, Ouémé Supérieur, Trois Rivières, Tchaourou et Ouénou-Bénou dans la région soudanienne au Bénin (figure 1), où la présence naturelle de $D$. mespiliformis (photo 3) a été fortement renseignée (Gnonlonfoun et al., 2015 ; Djagoun et al., 2010 ; Bonou et al., 2009). Les forêts classées de WariMaro, de l'Ouémé Supérieur et de Tchaourou sont davantage situées dans la commune de Tchaourou. La forêt classée de l'Alibori Supérieur se trouve dans la commune de Sinendé et celles de Ouénou-Bénou et de l'Ouémé Supérieur dans la commune de Bembèrèkè. Toutes ces communes sont sous l'influence d'un climat tropical du type soudanien humide, caractérisé par deux saisons de durée presque égale. En 2013, la population humaine y était en forte croissance (environ 4,6 \%) (INSAE, 2016). Le nombre d'habitants en 2013 était de 223138 à Tchaourou, 91672 à Sinendé et 131255 à Bembèrèkè (INSAE, 2016). Les principales catégories socioculturelles vivant dans ces communes sont constituées d'ethnies telles que les Bariba, les Nago, les Peuhls et les Wamma. Leurs principales activités sont la production agricole, l'élevage des ruminants et les petits commerces. Par ailleurs, ces groupes ethniques représentent les couches les plus pauvres du Bénin. Leurs dépenses journalières restent inférieures à 1 USD (INSAE, 2016). Par conséquent, elles dépendent fortement des fruitiers sauvages, utilisés à des fins alimentaires (humain et animal), sanitaires (humain), d'infrastructures et soins de santé pour les animaux (Vodouhê et al., 2009).

\section{Méthode d’échantillonnage}

L'échantillonnage dans cette étude a été réalisé en utilisant les données sociodémographiques des localités d'enquête (INSAE, 2016). Au total, 233 personnes ont été interviewées (139 hommes et 94 femmes) à leurs domiciles et dans les langues locales. Chaque catégorie sociodémographique a été proportionnellement représentée dans l'échantillon enquêté (tableau I). Celui-ci incluait les principales catégories socioprofessionnelles (agriculteurs ou producteurs agricoles, guérisseurs traditionnels ou tradipraticiens, éleveurs, artisans et commerçants). Les personnes enquêtées ont été sélectionnées en se basant également sur les classes d'âge (Assogbadjo et al., 2008) ainsi que sur la volonté de consacrer son temps à l'interview, la période de collecte des données coïncidant avec le moment des différentes activités des populations. L'âge minimal des personnes enquêtées était de 15 ans environ, car à cet âge l'individu est capable d'expliciter l'utilisation qu'il fait de l'espèce (IUCN, 2014).

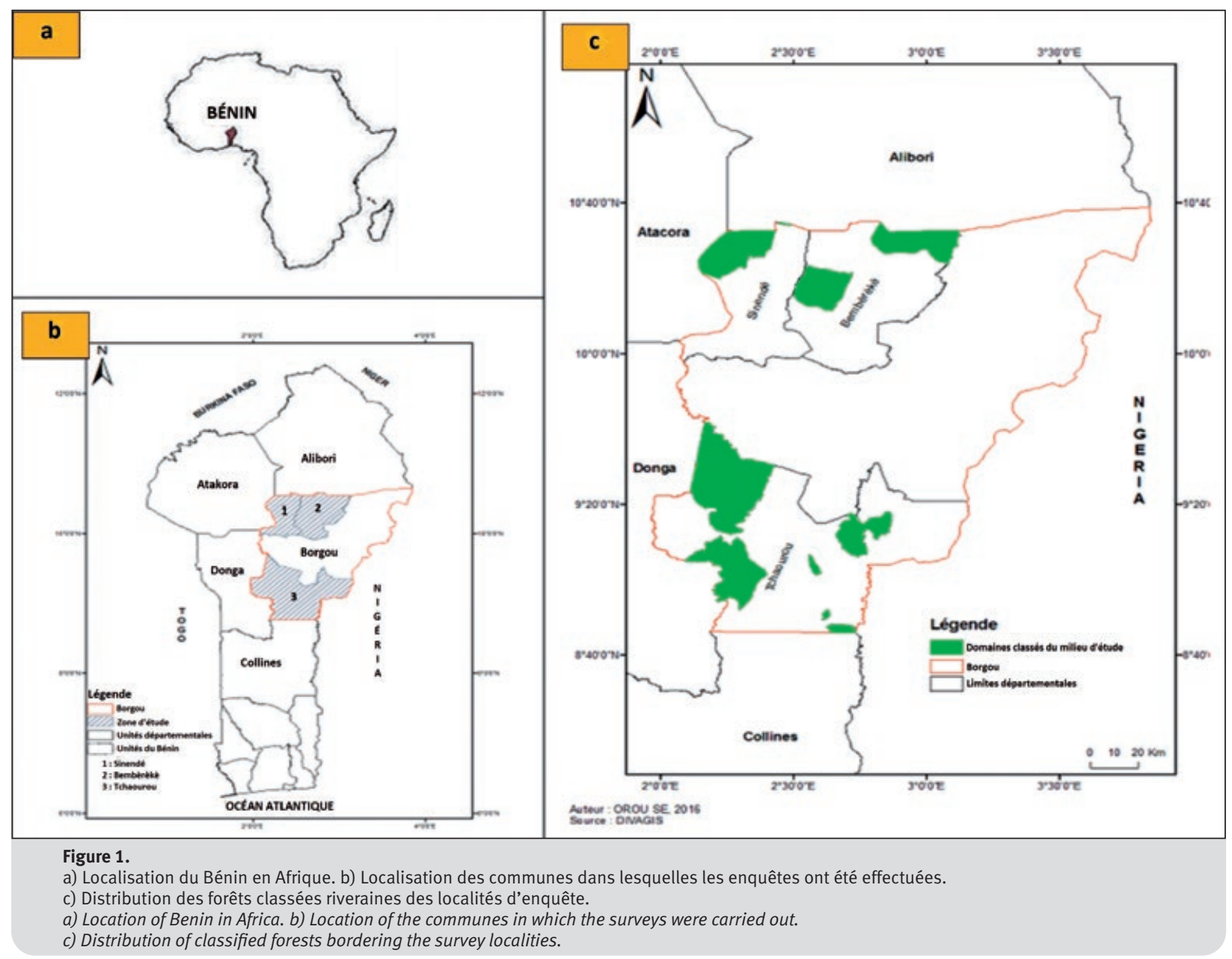




\section{Enquêtes ethnobotaniques}

Les données ont été collectées à travers les observations des participants et des entretiens individuels et semi-structurés. Les enquêtes ont été effectuées sous le couvert des personnes-ressources de chaque localité. La fiche de collecte de données utilisée a renseigné principalement sur l'âge, le sexe, la catégorie socioculturelle, la profession ou l'activité principale et la provenance de l'enquêté. Pour ce qui concerne l'espèce, la fiche a visé les informations sur ses usages et les organes utilisés. Les données concernant l'importance culturelle de la plante et ses organes ont été également renseignées lors des entretiens. La liste des catégories d'usages adoptée s'est inspirée des usages obtenus par Vodouhê et al. (2009) : alimentaire, bois de service et d'œuvre, bois énergie, fourrage, médecine et autres. Les scores d'importance culturelle des catégories d'usages de la plante et de ses organes ont été attribués par chaque interviewé en s'appuyant sur la grille de Belem et al. (2008). Ces scores ont varié de 0 à 3, avec : 0 pour la catégorie d'usages la moins importante ; 1 pour la catégorie relativement importante ; 2 pour celle importante ; 3 pour la catégorie d'usages très importante.

\section{Traitement et analyses des données}

Le traitement des informations a été consacré au calcul des indices ethnobotaniques usuels documentés dans les travaux de Assogba et al. (2017) et de Lougbegnon et al. (2015). Il s'agit de la fréquence relative de citation, la valeur des usages totaux rapportés, la valeur des usages rapportés par organe, l'indice d'importance culturelle.

La fréquence relative de citation est le nombre de fois qu'un usage spécifique de l'espèce a été cité par les interviewés d'une catégorie socioculturelle rapporté au nombre total des interviewés de la même catégorie socioculturelle. La valeur élevée de cet indice révèle généralement un accord commun pour le type d'usage ou organe/partie utilisé au sein de la catégorie socioculturelle.

Les valeurs des usages rapportés (totaux et par organe/ partie de la plante) représentent le nombre moyen d'usages spécifiques rapportés pour la plante et pour l'organe/partie de la plante dans chaque catégorie socioculturelle.

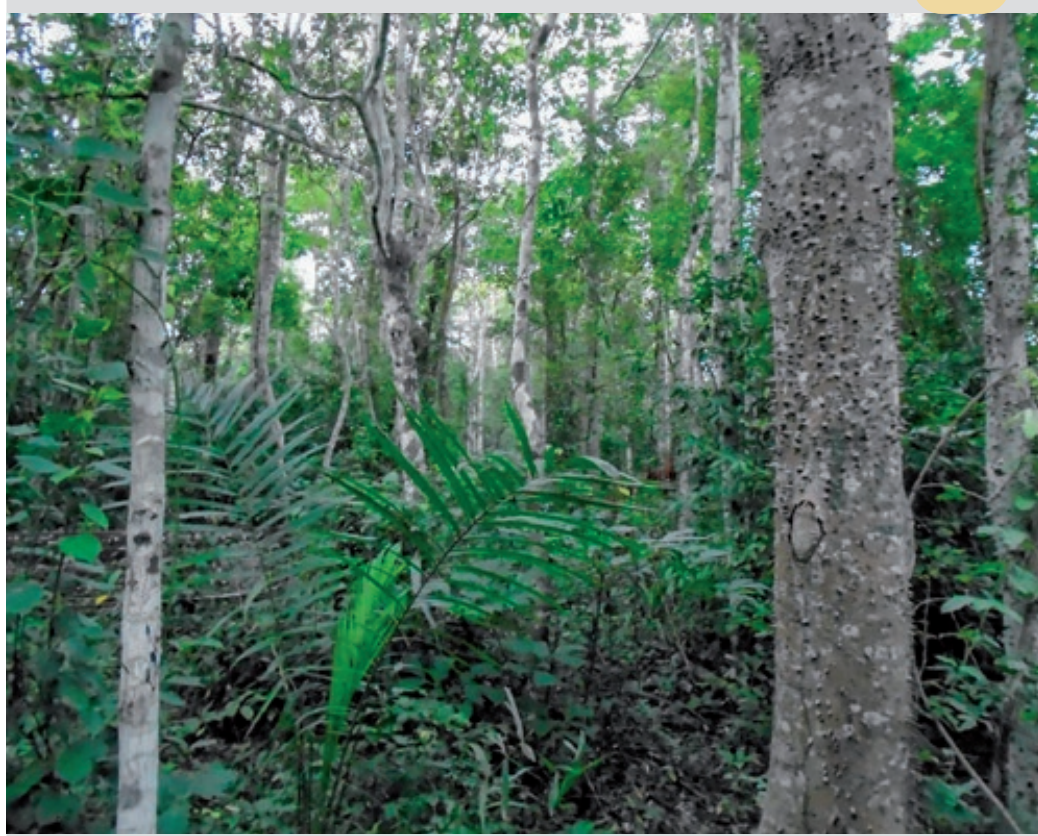

Photo 3.

Peuplement forestier à dominance de Diospyros mespiliformis. Forest stand dominated by Diospyros mespiliformis.

Photo A. Daanon.

L'indice d'importance se réfère à l'indice d'importance culturelle de l'espèce (Houehanou et al., 2011). Il indique, d'une part, la valeur culturelle attribuée à la plante et, d'autre part, celle rapportée aux organes de la plante par les populations. Ainsi, ces indices ont mis en exergue la catégorie d'usages et l'organe/partie de la plante les plus importants, selon les facteurs sociodémographiques (âge, sexe, catégorie socioculturelle, provenance socioculturelle et groupe socioprofessionnel).

Des matrices de données sur ces indices en relation avec ces facteurs sociodémographiques ont été construites. Une analyse de la covariance sur les usages totaux rapportés a été effectuée afin d'évaluer les déterminants sociodémographiques qui ont influencé l'utilisation de l'espèce. La réalisation d'une analyse en composantes principales a permis de caractériser les utilisations de l'espèce suivant ces déterminants.

Tableau I.

Effectif des personnes enquêtées selon la catégorie sociodémographique, ethnique et le genre. Number of people surveyed by socio-demographic category, ethnicity and gender.

\begin{tabular}{|c|c|c|c|c|c|c|c|c|c|}
\hline \multirow[t]{2}{*}{ Catégorie sociodémographique } & \multicolumn{2}{|c|}{ Bariba } & \multicolumn{2}{|c|}{ Nago } & \multicolumn{2}{|c|}{ Wamma } & \multicolumn{2}{|c|}{ Peuhls } & Total \\
\hline & $\mathbf{F}$ & H & $\mathbf{F}$ & $\mathbf{H}$ & $\mathbf{F}$ & $\mathbf{H}$ & $\mathbf{F}$ & $\mathbf{H}$ & \\
\hline Jeunes (âge < 30 ans) & 7 & 8 & 10 & 9 & 5 & 7 & 3 & 4 & 53 \\
\hline Adultes (30< âge <60) & 14 & 23 & 19 & 25 & 9 & 12 & 6 & 11 & 119 \\
\hline Vieux (âge $\geq 60$ ans) & 10 & 12 & 8 & 12 & 4 & 8 & 2 & 5 & 61 \\
\hline Total & 31 & 43 & 37 & 46 & 18 & 27 & 11 & 20 & 233 \\
\hline
\end{tabular}

$\mathrm{F}$ : femme ; $\mathrm{H}$ : homme. 
Tableau II.

Résultats de l'analyse de la covariance sur les usages totaux rapportés de Diospyros mespiliformis. Results of analysis of covariance on total reported uses of Diospyros mespiliformis.

\begin{tabular}{|c|c|c|c|c|c|}
\hline Source de variation & DL & SS & MS & F value & $\operatorname{Pr}(>F)$ \\
\hline Provenance & 3 & 0,96 & 0,32 & 0,258 & $0,8551^{\text {ns }}$ \\
\hline Catégorie socioculturelle & 8 & 12,02 & 1,503 & 1,213 & $0,3085^{\text {ns }}$ \\
\hline Sexe & 1 & 4,99 & 4,986 & 4,024 & $0,0497^{*}$ \\
\hline Profession & 4 & 0,95 & 0,237 & 0,191 & $0,9422^{\text {ns }}$ \\
\hline Âge & 1 & 5,47 & 5,469 & 4,414 & $0,0402^{*}$ \\
\hline Provenance $\times$ catégorie socioculturelle & 1 & 6,01 & 6,013 & 4,853 & $0,0317^{\star}$ \\
\hline Provenance $\times$ sexe & 1 & 1,37 & 1,37 & 1,106 & $0,2975^{\text {ns }}$ \\
\hline Catégorie socioculturelle $\times$ sexe & 3 & 5,36 & 1,786 & 1,442 & $0,2404^{\mathrm{ns}}$ \\
\hline Provenance $\times$ profession & 6 & 11,23 & 1,871 & 1,51 & $0,1916^{\mathrm{ns}}$ \\
\hline Catégorie socioculturelle $\times$ profession & 5 & 1,97 & 0,394 & 0,318 & $0,9003^{\text {ns }}$ \\
\hline Sexe $\times$ profession & 2 & 0,62 & 0,312 & 0,252 & $0,7781^{\text {ns }}$ \\
\hline Provenance $\times$ âge & 2 & 6,71 & 3,356 & 2,709 & $0,0754^{\text {ns }}$ \\
\hline Catégorie socioculturelle $\times$ âge & 5 & 9,71 & 1,942 & 1,567 & $0,1844^{\mathrm{ns}}$ \\
\hline Sexe $\times$ âge & 1 & 0,39 & 0,392 & 0,316 & $0,5762^{\text {ns }}$ \\
\hline Profession $\times$ âge & 3 & 7,5 & 2,501 & 2,019 & $0,1217^{\mathrm{ns}}$ \\
\hline Provenance $\times$ sexe $\times$ âge & 1 & 2,9 & 2,902 & 2,342 & $0,1315^{\text {ns }}$ \\
\hline Provenance $\times$ profession $\times$ âge & 3 & 5,87 & 1,956 & 1,578 & $0,2048^{\text {ns }}$ \\
\hline Résiduels & 56 & 69,39 & 1,239 & & \\
\hline
\end{tabular}

DL : degré de liberté ; SS : somme des carrés des écarts ; MS : carré moyen ; Fvalue : statistique de Fisher ; $\operatorname{Pr}(\gg F)$ : valeur de probabilité associée.

Codes du niveau de significativité $: 0,000={ }^{* \star \star} ; 0,001={ }^{* \star} ; 0,01={ }^{*} ;>0,05=$ non significatif (ns).

$\mathrm{X} \times \mathrm{Y}$ : interaction entre deux variables; $\mathrm{X} \times \mathrm{Y} \times \mathrm{Z}$ : interaction entre trois variables.

L'étude s'est également focalisée sur l'évaluation de l'importance des usages ethnobotaniques des organes de l'espèce suivant les catégories sociodémographiques. Une analyse de la covariance a été effectuée pour faire ressortir les déterminants (facteurs) sociodémographiques qui ont influencé l'utilisation des différents organes de l'espèce. Les valeurs des usages rapportés par organe ont été soumises à une analyse en composantes principales afin de déterminer les relations entre les différents usages des organes et les déterminants sociodémographiques. Auparavant, un modèle linéaire généralisé simplifié de la famille de Poisson (basé sur la valeur du critère d'information d'Akaike) a été retenu pour tester l'effet des classes d'âge sur l'utilisation des organes de l'espèce. Enfin, le même modèle linéaire généralisé de la famille de Poisson a été adopté pour tester la significativité de l'influence des autres utilisations de l'espèce sur ses usages alimentaires. Toutes les analyses ont été effectuées avec le logiciel R version 3.2 (R Core Team, 2016).

\section{Résultats}

\section{Utilisations faites de $D$. mespiliformis}

Les personnes enquêtées ont confirmé que les organes de l'espèce sont généralement collectés à des fins alimentaires et médicinales, mais aussi comme brosse végétale, fourrage, bois énergie, bois de service et bois d'œuvre. Les résultats de l'analyse de la covariance sur les usages totaux rapportés (tableau II) ont révélé que les usages de $D$. mespiliformis sont déterminés par l'âge, le sexe et la provenance des catégories socioculturelles $(P<0,05)$. En effet, il s'est révélé que $D$. mespiliformis est majoritairement utilisé par les hommes adultes.

Par ailleurs, la projection simultanée des catégories socioculturelles, de la provenance et des utilisations de l'espèce dans les plans d'axes factoriels (figure 2) a montré que les utilisations comme bois énergie et bois d'œuvre sont plus fréquentes chez les Peuhls de Tchaourou et les Bariba de Sinendé. Cependant, les Bariba de Tchaourou, de Sinendé et les Peuhls de Sinendé ont davantage utilisé $D$. mespiliformis à des fins alimentaires, comme fourrage et en pharmacopée. S'agissant des Peuhls et Wamma de Tchaourou et des Bariba de Bembèrèkè, l'espèce a été plus employée comme brosse végétale et bois de service. Notons que les Nago et les Peuhls de Bembèrèkè ont été moins intéressés par l'espèce. Ces résultats ont été expliqués par $67,64 \%$ des variabilités liées aux usages de $D$. mespiliformis au sein des catégories socioculturelles.

\section{Usages des organes de $D$. mespiliformis}

Les organes utilisés de $D$. mespiliformis ont été les fruits, les feuilles, les tiges, les écorces, les racines, les pulpes et les noix. L'importance culturelle de chaque organe de $D$. mespiliformis a varié significativement suivant les facteurs sociodémographiques. En effet, l'analyse de la cova- 

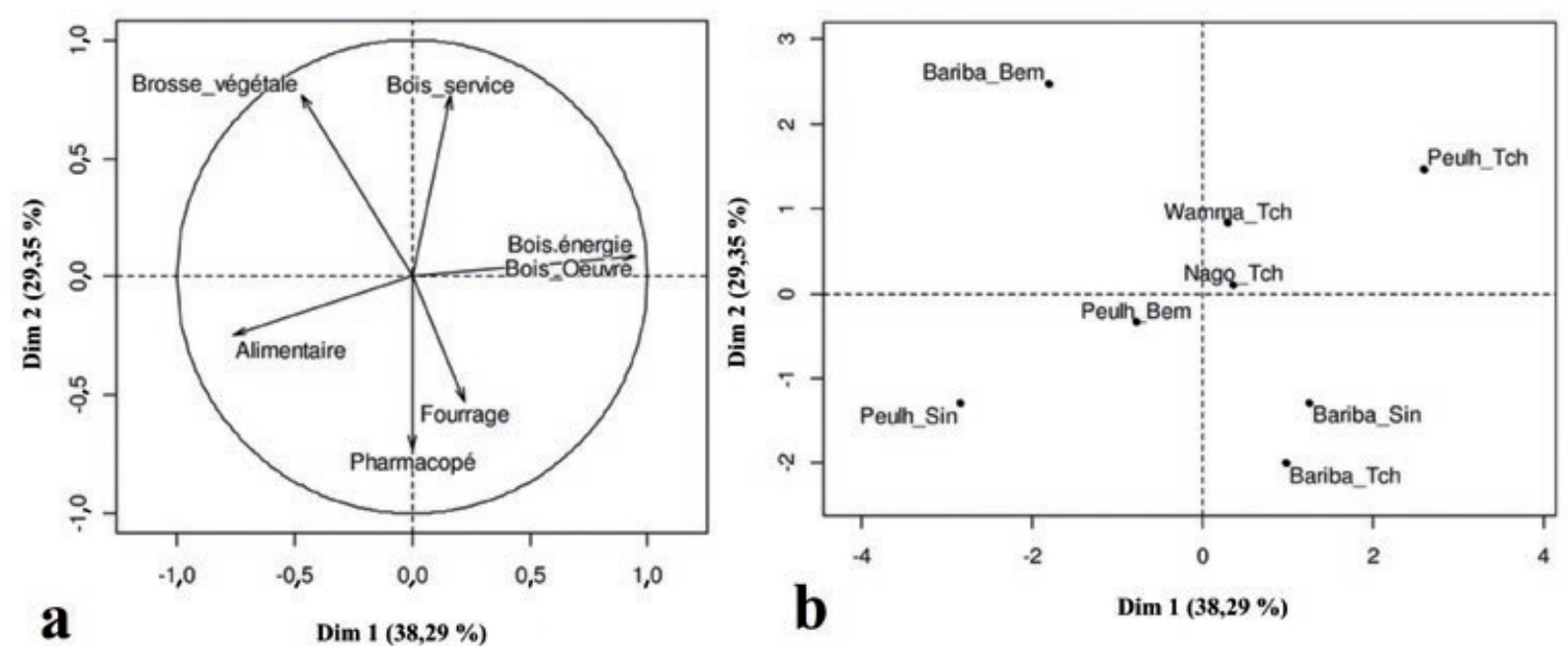

Figure 2.

Projection des usages de Diospyros mespiliformis (a) et des provenances socioculturelles utilisatrices de $D$. mespiliformis (b) dans le plan d'axe formé par les deux premières composantes principales.

Projection of Diospyros mespiliformis uses (a) and socio-cultural provenances using D. mespiliformis (b) in the axis plane formed by the first two principal components.

Peuhl Sin $=$ Peuhls de Sinendé $;$ Bariba $\sin =$ Bariba de Sinendé $;$ Peuhl Bem $=$ Peuhls de Bembèrèkè ;

Bariba_Bem = Bariba de Bembèrèkè $;$ Nago_Tch = Nago de Tchaourou ; Wamma_Tch = Wamma de Tchaourou ;

Peuhl_Tch = Peuhls de Tchaourou ; Bariba_Tch = Bariba de Tchaourou.

\section{Tableau III}

Résultats de l'analyse de la covariance sur les usages totaux rapportés des organes de Diospyros mespiliformis.

Results of the analysis of covariance on the total reported uses of Diospyros mespiliformis organs.

\begin{tabular}{|c|c|c|c|c|}
\hline Source de variation & DL & SS & MS & F value \\
\hline Provenance & 3 & 3,13 & 1,044 & $1,05 \mathrm{~ns}$ \\
\hline Catégorie socioculturelle & 8 & 6,94 & 0,867 & $0,872 \mathrm{~ns}$ \\
\hline Sexe & 1 & 3,13 & 3,125 & $3,142 \mathrm{~ns}$ \\
\hline Profession & 4 & 7,91 & 1,977 & $1,987 \mathrm{~ns}$ \\
\hline Âge & 1 & 4,97 & 4,965 & $4,992^{\star}$ \\
\hline Provenance $\times$ catégorie socioculturelle & 1 & 2,62 & 2,623 & $2,637 \mathrm{~ns}$ \\
\hline Provenance $\times$ sexe & 1 & 1,25 & 1,247 & $1,253 \mathrm{~ns}$ \\
\hline Catégorie socioculturelle $\times$ sexe & 3 & 3,85 & 1,285 & $1,291 \mathrm{~ns}$ \\
\hline Provenance $\times$ profession & 6 & 7,5 & 1,249 & $1,256 \mathrm{~ns}$ \\
\hline Catégorie socioculturelle $\times$ profession & 5 & 7,16 & 1,431 & 1,439 ns \\
\hline Sexe $\times$ profession & 2 & 0,4 & 0,198 & $0,199 \mathrm{~ns}$ \\
\hline Provenance $\times$ âge & 2 & 1,88 & 0,939 & $0,944 n s$ \\
\hline Catégorie socioculturelle $\times$ âge & 5 & 3,7 & 0,74 & $0,744 \mathrm{~ns}$ \\
\hline Sexe $\times$ âge & 1 & 0,06 & 0,057 & $0,057 \mathrm{~ns}$ \\
\hline Profession $\times$ âge & 3 & 3,1 & 1,032 & $1,037 \mathrm{~ns}$ \\
\hline Provenance $\times$ sexe $\times$ âge & 1 & 0,01 & 0,013 & $0,013 n s$ \\
\hline Provenance $\times$ profession $\times$ âge & 3 & 8,68 & 2,893 & $2,908^{*}$ \\
\hline
\end{tabular}

DL : degré de liberté ; SS : somme des carrés des écarts ; MS : carré moyen ; Fvalue : statistique de Fisher.

Codes du niveau de significativité : 0,05 $={ }^{*} ;>0,05=$ non significatif (ns). $X \times Y$ : interaction entre deux variables;

$\mathrm{X} \times \mathrm{Y} \times \mathrm{Z}$ : interaction entre trois variables. riance a montré que l'âge des enquêtés et les interactions provenance-profession-âge ont déterminé significativement $(P<0,05)$ le niveau d'usage des organes de $D$. mespiliformis par les populations (tableau III). Les racines et les écorces de la plante (photo $4 \mathrm{a}$ et b) ont été plus utilisées par toutes les classes d'âge (figure 3). Les racines le sont plus par les vieux suivis des adultes, tandis que le contraire a été obtenu pour les écorces. Cependant, les jeunes sont ceux qui ont le plus utilisé les fruits de l'espèce. Notons que les pulpes, les noix et les tiges le sont très peu dans le milieu d'étude (figure 3).

La projection simultanée de l'usage des organes de la plante et l'interaction provenance-profession-âge dans le plan des axes formés par les deux premières composantes principales $(63,67 \%$ de toute la variabilité) sont présentées dans la figure 4. Cette analyse a révélé que les racines, les tiges et les noix de $D$. mespiliformis (figure 4 a) sont les organes les plus recherchés par les éleveurs adultes de Bembèrèkè, les jeunes éleveurs de Sinendé, les vieux éleveurs de Tchaourou, les vieux agriculteurs de Tchaourou et les agriculteurs adultes de Tchaourou (figure 4b). Cependant, les écorces, les feuilles et les pulpes (figure 4a) sont, quant à elles, les parties les plus utilisées par les vieux agriculteurs de Bembèrèkè, les jeunes agriculteurs de Bembèrèkè et les commerçants adultes de Bembèrèkè et de Sinendé (figure 4b). Par ailleurs, les fruits et les feuilles (figure $4 a$ ) 
Bois et Forêts des Tropiques - ISSN: L-0006-579X

Volume $347-1^{\text {st }}$ quarter - February 2021 - p. 29-40

OCUS / DIOSPYROS MESPILIFORMIS USE

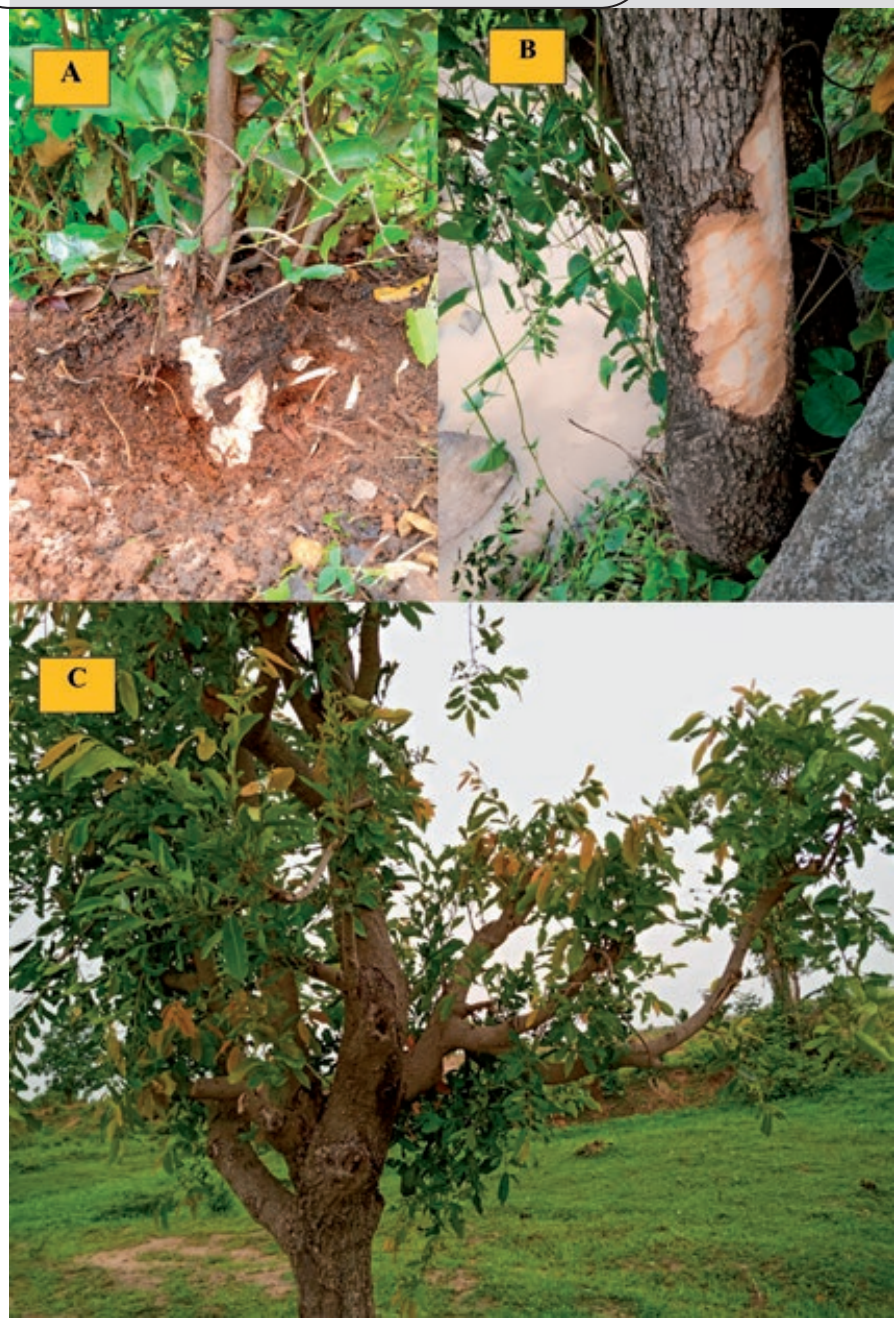

Photos 4.

Arbres de Diospyros mespiliformis dont les organes ont été récoltés:

A) racines; B) écorce de tronc; C) branches et feuilles.

Trees of Diospyros mespiliformis from which organs were collected:

A) roots; B) trunk bark; C) branches and leaves.

Photos B. A. Akakpo.

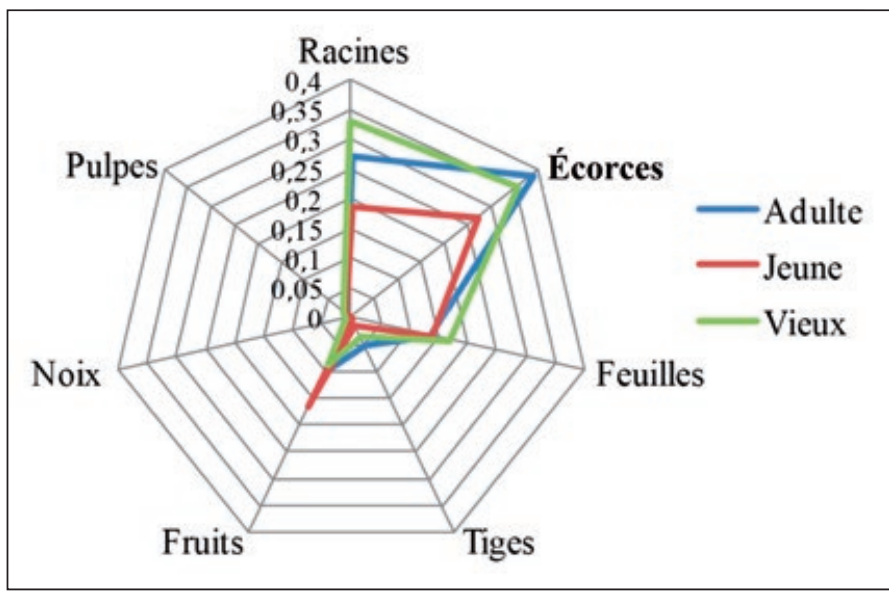

Figure 3.

Diagramme en radar montrant l'importance des organes utilisés de Diospyros mespiliformis suivant les classes d'âge.

Radar diagram showing the importance of the organs used of Diospyros mespiliformis according to age classes. intéressent davantage les jeunes éleveurs de Tchaourou, les éleveurs adultes de Bembèrèkè et les vieux éleveurs de Sinendé (figure 4b).

Les tiges et les feuilles (figure $5 \mathrm{a}$ ) sont plus fréquemment utilisées par les adultes et les vieux éleveurs de Tchaourou ainsi que les vieux agriculteurs de Bembèrèkè (figure 5b). Enfin, les fruits, les noix et les écorces (figure 5a) de $D$. mespiliformis ont été à Bembèrèkè préférés par les adultes commerçants et éleveurs, à Sinendé par les adultes commerçants et à Tchaourou par les vieux et les adultes agriculteurs (figure $5 b$ ).

\section{Influence des autres utilisations de $D$. mespiliformis sur le niveau d'usage alimentaire}

Le résultat de la régression linéaire généralisée de Poisson réalisée sur la matrice des valeurs des usages rapportés par les enquêtés est présenté dans le tableau IV. Les usages de l'espèce tels que le bois (œuvre et énergie) et le fourrage ont eu une influence très significative $(P<0,000)$ et négative sur l'usage alimentaire (fruit et pulpe) de l'espèce. En d'autres termes, les usages de l'espèce comme bois d'œuvre et énergie peuvent entraîner une baisse de sa valorisation en tant que produit alimentaire. En effet, une unité d'usage comme le bois énergie fait baisser la possibilité d'usage alimentaire de 0,86 lorsque les usages en tant que fourrage, bois d'œuvre et pharmacopée sont maintenus constants. Pour l'utilisation en fourrage, une unité de cet usage fait baisser la possibilité d'usage alimentaire de 3,97 lorsque les autres usages sont également restés constants (figure 3). Par contre, aucun impact significatif n'a été relevé en ce qui concerne les utilisations en tant que brosse végétale et bois de service sur la possibilité de l'usage alimentaire de l'espèce $(P>0,05)$. Toutefois, l'usage en pharmacopée traditionnelle n'est pas sans impact négatif sur la possibilité d'utiliser l'espèce en alimentation.

\section{Discussion}

Cette étude a montré que Diospyros mespiliformis est une espèce à usages multiples dans le Nord du Bénin. Son importance varie aussi suivant les catégories sociodémographiques des localités étudiées. Sept catégories d'usages y ont été rapportées. Il s'agit des usages alimentaires, en pharmacopée, comme bois d'œuvre, bois de service, bois énergie, brosse végétale et fourrage. Ces résultats confirment les travaux de Djagoun et al. (2010) et de Vodouhê et al. (2009), qui ont recensé pratiquement les mêmes utilisations au Bénin. Cependant, l'évaluation dans cette étude des variabilités des utilisations de l'espèce suivant les facteurs sociodémographiques reste la première de l'espèce au Bénin.

Les différentes utilisations de l'espèce et de ses organes sont déterminées par l'âge, le sexe, la provenance socioculturelle et la profession principale des utilisateurs. Les mêmes observations ont été faites dans plusieurs autres études au Bénin, dont celles de Wédjangnon et al. (2016) sur Mansonia altissima, de Akouehou et al. (2014) sur Artocarpus altilis et de Koura et al. (2011) sur Parkia biglobosa. Dans cette étude, ce sont les hommes adultes qui utilisent le 

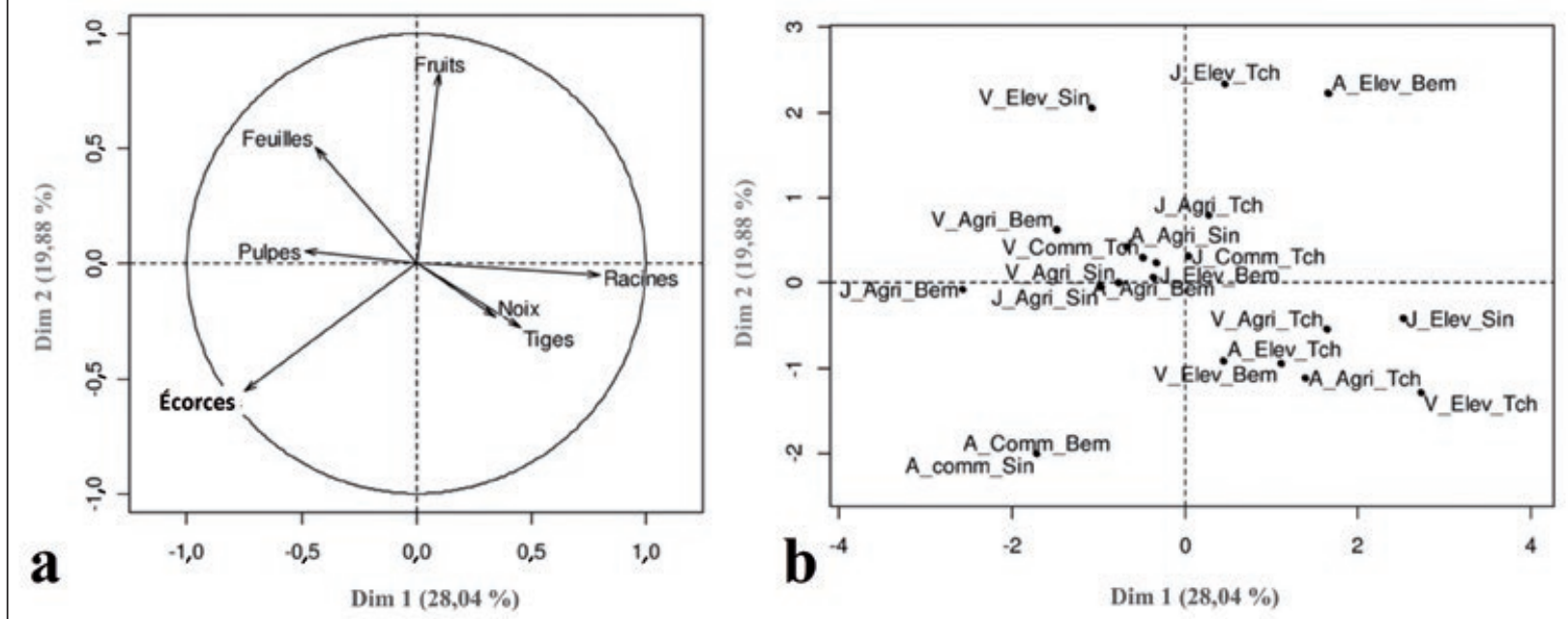

Figure 4.

Projection des organes utilisés de Diospyros mespiliformis (a) et de l'interaction âge x provenance x socioculturelle (b) dans le plan d'axe formé par les deux premières composantes principales.

Projection of the used organs of Diospyros mespiliformis (a) and the age x provenance x socio-cultural interaction (b) in the axis plane formed by the first two principal components axis plane formed by the first two principal components.

V_Elev_sin = vieil éleveur de Sinandé ; A_Comm_Sin = adulte commerçant de Sinandé ; J_Agri_Sin = jeune agriculteur de Sinandé ; A_Agri_Sin = adulte agriculteur de Sinandé ; V_Agri_Sin = vieil agriculteur de Sinandé ; J_Elev_sin = jeune éleveur de Sinandé ; A_Comm_Bem = adulte commerçant de Bembèrèkè ; J_Agri_Bem = jeune agriculteur de Bembèrèkè ; $A \_$Agri_Bem = adulte agriculteur de Bembèrèkè ; V_Elev_Bem = vieil éleveur de Bembèrèkè ; I Elev_Bem = jeune éleveur de Bembèrèkè ; $A$ Elev Bem = adulte éleveur de Bembèrèkè ; I Elev Tch = jeune éleveur de Tchaourou ; A_Elev_Tch = adulte éleveur de Tchaourou ; V_Elev_Tch = vieil éleveur de Tchaourou ; I_Agri_Tch = jeune agriculteur de Tchaourou ; A_Agri_Tch = adulte agriculteur de Tchaourou ; V_Agri_Tch = vieil agriculteur de Tchaourou ; I_Comm_Tch = jeune commerçant de Tchaourou ; V_Comm_Tch = vieux commerçant de Tchaourou.

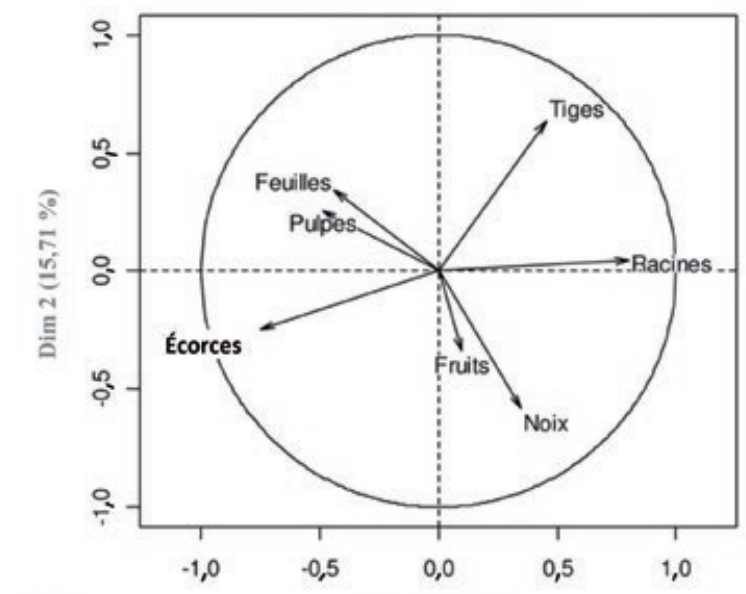

a

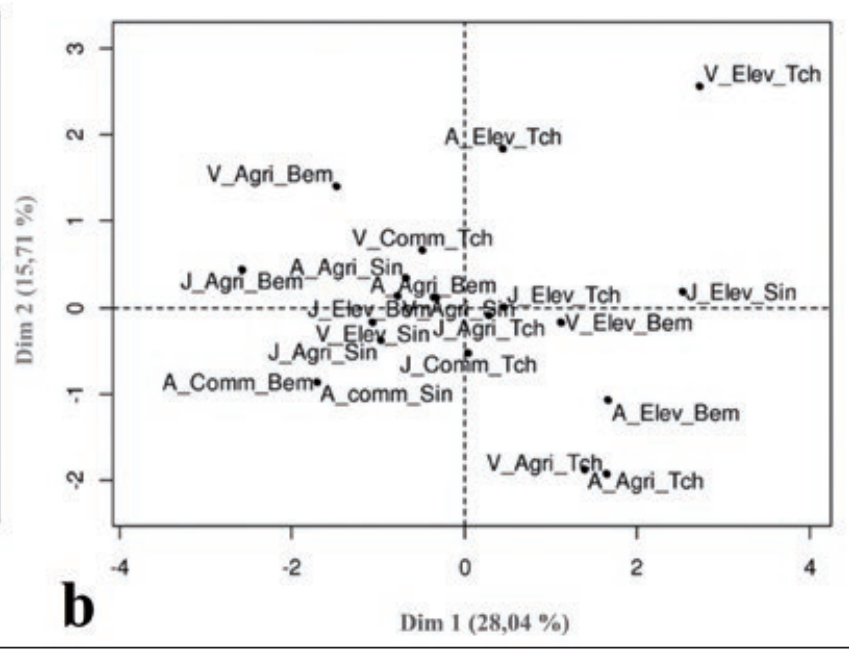

Figure 5.

Projection des organes utilisés de Diospyros mespiliformis (a) et de l'interaction âge x provenance x socioculturelle (b) dans le plan d'axe formé par les composantes principales 1 et 3.

Projection of the used organs of Diospyros mespiliformis (a) and the age x provenance $x$ socio-cultural interaction (b) in the axis plane formed by the first two principal componentsaxis plane formed by principal components 1 and 3 .

V_Elev_sin = vieil éleveur de Sinandé ; A_Comm_Sin = adulte commerçant de Sinandé ; J_Agri_Sin = jeune agriculteur de Sinandé ; A_Agri_Sin = adulte agriculteur de Sinandé ; V_Agri_Sin = vieil agriculteur de Sinandé ; J_Elev_sin = jeune éleveur de Sinandé ; A_Comm_Bem = adulte commerçant de Bembèrèkè ; J_Agri_Bem = jeune agriculteur de Bembèrèkè ; $A \_$Agri_Bem = adulte agriculteur de Bembèrèkè ; V_Elev_Bem = vieil éleveur de Bembèrèkè ; J_Elev_Bem = jeune éleveur de Bembèrèkè ; $A \_E l e v \_B e m=$ adulte éleveur de Bembèrèkè ; J_Elev_Tch = jeune éleveur de Tchaourou ; A_Elev_Tch = adulte éleveur de Tchaourou ; V_Elev_Tch = vieil éleveur de Tchaourou ; I_Agri_Tch = jeune agriculteur de Tchaourou ; A_Agri_Tch = adulte agriculteur de Tchaourou ; V_Agri_Tch = vieil agriculteur de Tchaourou ; I_Comm_Tch = jeune commerçant de Tchaourou ; V_Comm_Tch = vieux commerçant de Tchaourou.

plus l'espèce. Ces résultats peuvent se justifier par l'importance que cette catégorie sociodémographique accorde à la plante (Gbémavo et al., 2014). Cela peut être aussi dû au fait que, dans le milieu d'étude, les utilisations et la récolte des organes de la plante incombent plutôt aux hommes (Issifou et al., 2018) et ceux-ci dans une classe d'âge plus active. En effet, toutes les actions pour une bonne valorisation de 


\begin{abstract}
Tableau IV.
Résultat de la régression linéaire généralisée de Poisson sur les usages rapportés indiquant l'impact des catégories d'usages de Diospyros mespiliformis sur son utilisation alimentaire.

Result of the generalized linear Poisson regression on reported uses indicating the impact of the use categories of Diospyros mespiliformis on its food use.
\end{abstract}

\begin{tabular}{l|c|c|c} 
Usages & Estimateur & Std. Error & Z-value \\
\hline Intercept & $-0,01$ & 0,10 & $-0,10 \mathrm{~ns}$ \\
\hline Bois énergie & $-0,86$ & 0,18 & $-4,82^{\star \star \star}$ \\
\hline Bois d'œuvre & $-1,77$ & 0,25 & $-6,96^{\star \star \star}$ \\
\hline Bois de service & $-0,23$ & 0,15 & $-1,59 \mathrm{~ns}$ \\
\hline Brosse végétale & $-0,15$ & 0,14 & $-1,07 \mathrm{~ns}$ \\
\hline Fourrage & $-3,97$ & 0,71 & $-5,56^{\star \star \star}$ \\
\hline Pharmacopée & $-0,43$ & 0,15 & $-2,78^{\star \star}$
\end{tabular}

Std. Error : erreur standard ; Z-value : statistique calculée. Codes du niveau de significativité : $0,000=* \star \star$; $0,001={ }^{\star \star} ; 0,01={ }^{\star} ;>0,05=$ non significatif (ns).

pays, des zones adjacentes peuvent ne pas faire les mêmes usages des ressources. Cette variation qui semblerait non négligeable dans les décisions de valorisation durable de l'espèce pourrait s'expliquer par les différences culturelles entre catégories socioculturelles (Assogba et al., 2017). Cependant, une différenciation dans les usages et organes utilisés a été observée à l'intérieur d'une même catégorie socioculturelle géographiquement séparée. C'est le cas des Peuhls de Tchaourou et de Sinendé qui utilisent respectivement l'espèce comme bois (énergie et œuvre) et à des fins alimentaires. Ce résultat peut s'expliquer par l'émergence de nouvelles connaissances sur l'utilisation de l'espèce en raison de l'adaptation à la situation locale.

La profession des enquêtés influe aussi sur les catégories d'utilisations des organes de la plante. En effet, comme l'ont conclu Byg et Balslev (2004), les connaissances ethnobotaniques sont globalement influencées par les facteurs tels que l'ethnie, l'âge, le sexe, la religion, le niveau d'instruction, la position géographique et la profession de l'enquêté. Ainsi, l'importance des activités principales dans les variations des parties utilisées de $D$. mespiliformis peut être justifiée par l'activité de l'utilisateur. Par conséquent, les agriculteurs et les éleveurs récoltent plutôt les racines et les feuilles de la plante pour renforcer leur santé après les activités intenses de labour ou de pâture. Les tiges et les troncs sont principalement utilisés pour le bois énergie ou le bois d'œuvre par les agriculteurs car ces derniers représentent majoritairement les propriétaires du foncier dans les milieux enquêtés, alors que, chez les commerçants, les fruits, les noix et les pulpes de l'espèce sont activement recherchés ; cela peut aussi révéler le caractère marchand de ces organes de l'espèce. Les agriculteurs et les éleveurs qui les utilisent le font davantage à des fins de consommation circonstancielle.
Les usages comme bois (énergie et œuvre), fourrage et pharmacopée réduisent significativement la possibilité d'usage alimentaire de l'espèce. En effet, le recours à l'espèce en tant que bois (énergie et œuvre) signifie indirectement son élimination (Cunningham, 1996), par coupe ou par dévitalisation. Ce qui impacterait sa disponibilité pour des fins alimentaires et même constitue une menace pour sa valorisation durable. L'émondage de l'espèce pour le fourrage affecte aussi le cycle de reproduction de l'espèce (Gandon, 2003 ; Depommier, 1998) et donc sa potentialité pour des usages alimentaires. En ce qui concerne les usages en pharmacopée, les racines et les écorces de l'espèce sont recherchées par les populations. Ainsi, selon Belem et al. (2008) et Betti (2001), la récolte intensive des racines et des écorces des arbres peut entraîner la perte de leur vigueur et des troubles physiologiques. Du coup, la production de fruits est aussi touchée et impacterait directement l'usage de l'espèce à des fins alimentaires.

\section{Implication pour la valorisation et la conservation de $D$. mespiliformis}

L'espèce D. mespiliformis est principalement exploitée par les hommes adultes à des fins alimentaires, en pharmacopée, comme bois d'œuvre, bois de service, bois énergie, brosse végétale et fourrage dans le milieu d'étude. Des actions de valorisation et de conservation de l'espèce doivent maintenant être menées principalement en direction des hommes adultes des différentes communes considérées dans cette étude. La valorisation de l'espèce pour des usages tels que le bois énergie et le bois d'œuvre doit concerner plus particulièrement les Peuhls de Tchaourou et les Bariba de Sinendé, qu'ils soient agriculteurs ou éleveurs. Cependant, et considérant le fait que ces usages affectent non seulement l'usage alimentaire mais aussi la survie de l'espèce, des études doivent être menées afin de déterminer la productivité et la possibilité d'exploitation de l'espèce dans le milieu d'étude. En outre, des efforts de domestication doivent être déployés pour assurer sa conservation.

Les usages de l'espèce à des fins alimentaires, comme fourrage et en pharmacopée sont plus importants chez les Bariba de Tchaourou, de Sinendé et les Peuhls de Sinendé. Le renforcement des capacités de ces groupes socioculturels pour ces utilisations à travers des pratiques durables de collecte des organes sont nécessaires pour une meilleure conservation de l'espèce. Les racines et les écorces ont été les plus utilisées par toutes les classes d'âge de la population ; ce sont des organes vitaux pour l'espèce dont l'exploitation mettrait en danger sa survie. Il serait intéressant d'étudier la disponibilité des substances actives recherchées dans les racines et écorces, dans les feuilles et fruits, afin d'en créer des substituts. Il est donc maintenant urgent d'élaborer une stratégie de valorisation et de conservation de l'espèce $D$. mespiliformis dans le Nord du Bénin, afin de satisfaire les besoins des différents groupes socioculturels tout en pérennisant la disponibilité et la survie de l'espèce. 


\section{Conclusion}

De la présente étude, on peut conclure que Diospyros mespiliformis Hochst. est utilisée de plusieurs manières par les populations locales du Nord du Bénin. Les utilisations sollicitées de ses différents organes sont diversifiées et dépendent des groupes socioculturels et socioprofessionnels, des âges, des milieux de provenance des enquêtés et de leurs interactions. Les racines et les écorces sont les organes principalement prisés par les adultes et les vieux. Les Peuhls et les Bariba sont les groupes socioculturels qui constituent les principaux utilisateurs des différents organes de l'espèce. À cet effet, les Peuhls éleveurs sollicitent davantage les organes de l'espèce dans l'alimentation et la pharmacopée, tandis que les Bariba les utilisent plus en pharmacopée, comme brosse végétale et comme fourrage. Quant aux Nago et Wamma, ils s'attachent aux parties ligneuses de l'espèce pour le bois (service, énergie et œuvre). La convergence entre les organes les plus utilisés de l'espèce et les groupes professionnels révèle que les éleveurs et agriculteurs préfèrent les parties sensibles (racines et écorces) de l'espèce. La commercialisation de la plante se fait sans préférence particulière des organes. Pour une stratégie de valorisation et de conservation de l'espèce, non seulement ces conclusions sont à prendre en compte mais il faudra aussi évaluer les pressions de prélèvement pour chaque catégorie d'usages et les possibilités de domestication de l'espèce dans les systèmes agroforestiers (photo 5).

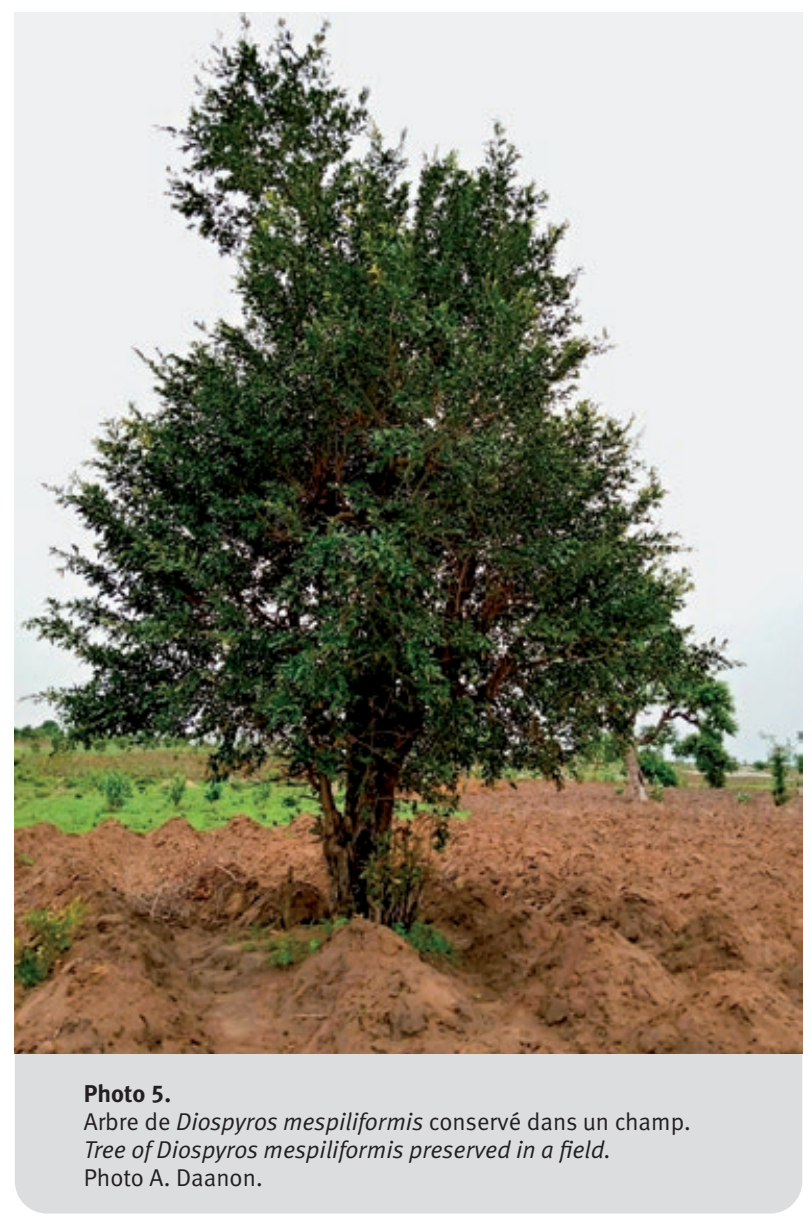

\section{Remerciements}

Les auteurs tiennent à remercier les populations pour leur disponibilité lors des collectes de données d'enquête et pour leur collaboration pertinente dans l'accomplissement de cette recherche.

\section{Références}

Akouehou G. S., Goussanou C. A., Idohou R., Dissou F. E., Azokpota P., 2014. Importance socioculturelle de Artocarpus altilis (Parkinson) Fosberg (Moraceae) au Sud-Bénin. Journal of Applied Biosciences, 75 (1) : 6173-6182. https://doi.org/10.4314/jab.v75i1.5 Arbonnier M., 2002. Trees, shrubs and lianas of West African dry zones. Versailles, France, Éditions Quæ, 573 p.

Assogba G. A., Fandohan A. B., Salako V. K., Assogbadjo A. E., 2017. Usages de Bombax costatum (Malvaceae) dans les terroirs riverains de la Réserve de biosphère de la Pendjari, République du Bénin. Bois et Forêts des Tropiques, $333: 17-29$. https://doi. org/10.19182/bft2017.333.a31465

Assogbadjo A. E., Amadji G., Glèlè Kakaï R., Mama A., Sinsin B., Van Damme P., 2009. Évaluation écologique et ethnobotanique de Jatropha curcas L. au Bénin. International Journal of Biological and Chemical Sciences, 3 (5) : 1065-1077. https://doi.org/10.4314/ iibcs.v3i5.51085

Assogbadjo A. E., Glèlè Kakaï R., Chadaré F. J., Thomson L., Kyndt T., Sinsin B., et al., 2008. Folk classification, perception and preferences of baobab products in West Africa: Consequences for species conservation and improvement. Economic Botany, 62 (1): 74-84. https://doi.org/10.1007/s12231-007-9003-6

Avocèvou-Ayisso C., Avohou T. H., Oumorou M., Dossou G., Sinsin B., 2012. Ethnobotany of Pentadesma butyracea in Benin: A quantitative approach. Ethnobotany Research and Applications, 10: 151-166. http://ethnobotanyjournal.org/index.php/era/article/ view/447

Belem B., Smith O. C., Theilade I., Bellefontaine R., Guinko S., Lykke A. M., et al., 2008. Identification des arbres hors forêt préférés des populations du Sanmatenga (Burkina Faso). Bois et Forêts des Tropiques, 298 (4) : 53-64. https://revues.cirad.fr/index.php/BF/ article/view/20366

Betti J. L., 2001. Vulnérabilité des plantes utilisées comme antipaludiques dans l'arrondissement de Mintom au sud de la réserve de biosphère du Dja (Cameroun). Systematics and Geography of Plants, 71 (2) : 661- 678. https://doi.org/10.2307/3668709

Bonnet P., Arbonnier M., Grard P., 2008. Ligneux du Sahel : outil graphique d'identification. Versailles, France, Éditions Quæ, CD-Rom. https://agritrop.cirad.fr/545941/

Bonou W., Glèlè Kakaï R., Assogbadjo A. E., Fonton H. N., Sinsin B., 2009. Characterization of Afzelia africana Sm. habitat in the Lama Forest reserve of Benin. Forest Ecology and Management, 258 (7): 1084-1092. https://doi.org/10.1016/i.foreco.2009.05.032

Byg A., Balslev H., 2004. Factors affecting local knowledge of palms in Nangaritza Valley, Southeastern Ecuador. Journal of Ethnobotany, 24 (2): 255-278. https://www.biodiversitylibrary.org/ page/32740564\#page/91/mode/1up

Codjia J. T. C., Assogbadjo A. E., Ekué M., 2003. Diversité et valorisation au niveau local des ressources végétales forestières alimentaires du Bénin. Cahiers Agricultures, 12 (5) : 321-331. https:// revues.cirad.fr/index.php/cahiers-agricultures/article/view/30405 Cunningham A. B., 1996. Peuples, parc et plantes. Recommandations pour les zones à usages multiples et les alternatives de développement autour du parc national de Bwindi Impénétrable, Ouganda. Paris, France, UNESCO, Document de travail Peuples et Plantes $\mathrm{n}^{\circ} 4,64 \mathrm{p}$. http://www.ethnopharmacologia.org/ prelude2018/pdf/biblio-hc-11-cunningham.pdf

Depommier D., 1998. Étude phénologique de Faidherbia albida. Effet de l'émondage, du site et de la dimension de l'arbre sur les phénophases de l'espèce au Burkina Faso. In : Campa C., Grignon C., Gueye M., Hamon S. (éds). L'acacia au Sénégal. Paris, France, ORSTOM/ISRA, coll. Colloques et séminaires, 159-179. https:// agritrop.cirad.fr/465566/ 
Diarra N., Togola A., Denou A., Willcox M., Daou C., Diallo D., 2016. Étude ethnobotanique des plantes alimentaires utilisées en période de soudure dans les régions Sud du Mali. International Journal of Biological and Chemical Sciences, 10 (1) : 184-197. https://doi. org/10.4314/ijbcs.v10i1.14

Djagoun C. A. M. S., Glèlè Kakaï R., Konnon D., Sewade C., Kouton M., Bonou W., et al., 2010. Potential Food and Medicinal Use of Plant Resources of the Ouémé Supérieur and N'Dali Classified Forests (Northern Benin). Fruit, Vegetable and Cereal Science and Biotechnology, 4 (1): 47-54.

Dossou-Yovo H. O., Vodouhé F. G., Sinsin B., 2014. Assessment of the medicinal uses of plant species found on termitaria in the Pendjari biosphere reserve in Benin. Journal of Medicinal Plant Research, 8 (8): 368-377. https://doi.org/10.5897/JMPR10.124

Ekué M. R., Sinsin B., Eyog-Matig O., Finkeldey R., 2010. Uses, traditional management, perception of variation and preferences in ackee (Blighia sapida K.D. Koenig) fruit traits in Benin: implications for domestication and conservation. Journal of Ethnobiology and Ethnomedicine, 6: 1-12. https://doi.org/10.1186/1746-4269-6-12 Fandohan B., Assogbadjo A. E., Glèlè Kakaï R., Kyndt T., De Caluwé E., Codjia J. T. C., Sinsin B., 2010. Women's traditional knowledge, use value, and the contribution of tamarind (Tamarindus indica L.) to rural households' cash income in Benin. Economic Botany, 64: 248-259. https://doi.org/10.1007/s12231-010-9123-2

Gandon B., 2003. L'émondage du Pterocarpus erinaceus (vène) : étude des pratiques et de leurs impacts sur l'arbre, sur 4 terroirs agro-sylvo-pastoraux du Sénégal Oriental. Mémoire DESS, Université de Paris-Val-de-Marne, France, 80 p. https://agritrop.cirad. fr $/ 528431$

Gbémavo C. J., Gnanglé C. P., Assogbadjo E. A., Glèlè Kakaï R. L., 2014. Analyse des perceptions locales et des facteurs déterminant l'utilisation des organes et des produits du Jatropha curcas Linn. (Euphorbiaceae) au Bénin. Agronomie Africaine, 26 (1) : 69-79. https://www.ajol.info/index.php/aga/article/view/104853

Gnonlonfoun I., Glèlè Kakaï R. L., Salako V. K., Assogbadjo A. E., Ouédraogo A., 2015. Structural analysis of regeneration in tropical dense forest: combined effect of plot and spatial distribution patterns. Acta Botanica Gallica: Botany Letters, 162 (1): 79-87.

Gouwakinnou G. N., Lykke A. M., Assogbadjo A. E., Sinsin B., 2011. Local knowledge, pattern and diversity of use of Sclerocarya birrea. Journal of Ethnobiology and Ethnomedicine, 7 (1): 8. https://doi.org /10.1080/12538078.2014.984332

Hoffman B., Gallaher T., 2007. Importance indices in ethnobotany. Ethnobotany Research and Applications, 5 (1): 201-218. http://ethnobotanyjournal.org/index.php/era/article/view/130

Houehanou T. D., Assogbadjo A. E., Kakaï R. G., Houinato M., Sinsin B., 2011. Valuation of local preferred uses and traditional ecological knowledge in relation to three multipurpose tree species in Benin (West Africa). Forest Policy and Economics, 13 (7): 554-562. https://doi.org/10.1016/i.forpol.2011.05.013

Idohou R., Assogbadjo A. E., Fandohan B., Gouwakinnou G. N., Glèlè Kakaï R. L., Sinsin B., et al., 2013. National inventory and prioritization of crop wild relatives: case study for Benin. Genetical Resources and Crop Evolution, 60 (4): 1337-1352. https://doi.org/10.1007/ s10722-012-9923-6

INSAE, 2016. Effectifs de la population des villages et quartiers de villes du Bénin. Rapport final, RGPH-Recensement Général de la Population et l'Habitat $4^{\mathrm{e}}$ édition. Cotonou, Bénin, Institut national de la statistique et de l'analyse économique, $85 \mathrm{p}$.

IUCN (International Union for Conservation of Nature), 2014. IUCN Evaluations of Nominations of Natural and Mixed Properties to the World Heritage List. Report to the World Heritage Committee TwentySixth Session, 24-29 June 2002, Budapest, Hungary. UNESCO, World Heritage Convention, 148 p. https://whc.unesco.org/ archive/2014/whc14-38com-inf8B2-en.pdf

Issifou I. S., Kpérkouma W. A., Dourma M., Atakpama W., Kanda M., Akpagana K., 2018. Valeur ethnobotanique de l'espèce Khaya senegalensis (Desr.) A. Juss. (meliaceae) auprès des populations riveraines de la chaîne de l'Atacora au Togo. Revue Marocaine des Sciences Agronomiques et Vétérinaires, 6 (1) : 64-72. https://www. agrimaroc.org/index.php/Actes IAVH2/article/view/512/570
Koura K., Ganglo J. C., Assogbadjo A. E., Agbangla C., 2011. Ethnic differences in use values and use patterns of Parkia biglobosa in Northern Benin. Journal of Ethnobiology and Ethnomedicine, 7 (42): 1-12. https://doi.org/10.1186/1746-4269-7-42

Lougbegnon T. O., Nassi K. M., Gbesso G. F., 2015. Ethnobotanique quantitative de l'usage de Chrysophyllum albidum G. Don par les populations locales au Bénin. Journal of Applied Biosciences, 95 : 9028-9038. https://doi.org/10.4314/jab.v95i1.12

Mapongmetsem P. M., Kapchie V. N., Tefempa B. H., 2012. Diversity of local fruit trees and their contribution in sustaining the rural livelihood in the Northern Cameroon. Ethiopian Journal of Environmental Studies and Management, 5 (1): 32-46. https://doi.org/10.4314/ ejesm.v5i1.5

Maxted N., Kell S. P., Ford-Lloyd B., 2008. Crop wild relatives: catalogues, threats, conservation and use. In: Maxted N., Dulloo E., FordLloyd B., Kell S. P., Iriondo J. M. (eds). Crop Wild Relatives Conservation and Use. Wallingford, United Kingdom, CAB International, 3-30. R Core Team, 2016. R: A language and environment for statistical computing. Vienna, Austria, R Foundation for Statistical Computing. https://www.R-project.org/

Vodouhê F. G., Coulibaly O., Greene C., Sinsin B., 2009. Estimating the local value of non-timber forest products to Pendjari biosphere reserve dwellers in Benin. Economic Botany, 63 (4): 397-412. https://doi.org/10.1007/s12231-009-9102-7

Wédjangnon A. A., Houètchégnon T., Ouinsavi C., 2016. Caractéristiques ethnobotaniques et importance socio-culturelle de Mansonia altissima A. Chev. au Bénin, Afrique de l'Ouest. Journal of Animal \& Plant Sciences, 29 (3) : 4678-4690. http://www.m.elewa.org/ IAPS/2016/29.3/4.Wedjangnon.pdf

\begin{tabular}{|c|c|}
\hline Rôle du contributeur & Noms des auteurs \\
\hline Conceptualisation & A. Daanon, M. Houinato \\
\hline Gestion des données & A. Daanon, E. A. Padonou, B. A. Akakpo \\
\hline Analyse formelle & A. Daanon, E. A. Padonou, B. A. Akakpo \\
\hline $\begin{array}{l}\text { Acquisition du } \\
\text { financement }\end{array}$ & A. Daanon \\
\hline $\begin{array}{l}\text { Enquête et } \\
\text { investigation }\end{array}$ & A. Daanon, B. A. Akakpo \\
\hline Méthodologie & M. Houinato, E. A. Padonou \\
\hline Gestion de projet & E. A. Padonou, B. A. Akakpo \\
\hline Ressources & E. A Padonou, A. Daanon \\
\hline Logiciels & $\begin{array}{l}\text { E. A. Padonou, A. Daanon, } \\
\text { B. A. Akakpo }\end{array}$ \\
\hline Supervision & E. A. Padonou, M. Houinato \\
\hline Validation & $\begin{array}{l}\text { E. A. Padonou, B. A. Akakpo, } \\
\text { A. Daanon, M. Houinato }\end{array}$ \\
\hline Visualisation & B. A. Akakpo, E. A. Padonou \\
\hline $\begin{array}{l}\text { Écriture - Préparation } \\
\text { de l'ébauche originale }\end{array}$ & $\begin{array}{l}\text { B. A. Akakpo, E. A. Padonou, } \\
\text { A. Daanon }\end{array}$ \\
\hline
\end{tabular}

Bois et Forêts des Tropiques - Revue scientifique du Cirad (c) Bois et Forêts des Tropiques (c) Cirad

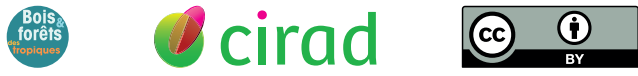

Cirad - Campus international de Baillarguet, 34398 Montpellier Cedex 5, France - Contact : bft@cirad.fr - ISSN : L-0006-579X 